Introduction Lorenz curves, while developed to measure income inequality, can describe the unequal distribution of sexual contacts in a population. Sexual partnerships are typically skewed with most individuals reporting few and some reporting many, but the degree of concentration is poorly understand. A better understanding has important implications for HIV and STI prevention, care and research, notably targeted PrEP and test-and-treat.

Methods We analysed data collected from an ongoing national HIV behavioural surveillance programme among gay and bisexual men (GBM) conducted in offline and online community settings in 2014. Participation was voluntary, anonymous and self-reported. Data were collected on the number of condomless anal intercourse partnerships (CAIPs) in the previous six months. We aimed to (i) describe the distribution of CAIPs; (ii) measure the concentration of CAIPs using Lorenz curves; (iii) identify potential "core" groups and compare their characteristics to other GBM.

Results Of 3027 GBM responding, 1575 (52\%) reported at least one CAIP. Of these 1575, the median, mean and range was 1, 3.5 and 1-250 CAIPs, and the distribution was skewed with $90 \%, 95 \%$ and $99 \%$ percentiles being $\leq 6, \leq 10$ and $\leq 39$ CAIPS respectively. An aggregate 5525 CAIPs were reported, with $9 \%$ of GBM accounting for 52\% of all CAIPs (those reporting >6 CAIPs); $5 \%$ accounting for $40 \%$ of all CAIPs (those reporting $>10$ CAIPs); and 1\% accounting for 19\% of all CAIPs (those reporting $40+$ CAIPs). The Gini index was 0.59 indicating high inequality. GBM in these three "cores" were more likely to be older, diagnosed HIV positive, have recent STI diagnoses and negative attitudes towards condoms and safe sex obligations.

Conclusion A large volume of condomless sex partnerships in this community sample was generated by a small proportion of GBM representing highly sexually connected nodes. Clinically, interventions with these GBM such as PrEP and early diagnosis and treatment can disrupt transmission of HIV and STIs across sexual networks and must be attractive and accessible to them.

\section{P4.85 YOUNG FEMALE GARMENT WORKERS IN MIGRATION, SEX WORK AND HIV: A STUDY OF MUMBAI CITY}

Prashika. International Institution for Population Sciences, Mumbai, India

\subsection{6/sextrans-2017-053264.580}

Introduction HIV/AIDS has become a major public health problem in India. Garment industry is one of the fastest growing industries attracting adolescents and young women. Since many risk behaviours, associated with transmission of HIV, are adopted in young age, it is very important to target the prevention efforts in the young age group. One of the major obstacles in conducting any health education campaign in this industries that, many workers shift from factory for better facilities. Hence governments as well as NGOs have failed to conduct HIV/AIDS awareness programmers in these industries. This has created a situation where in young workers lack proper knowledge about of HIV/AIDS, and also risky behaviour associated with it. To evaluate awareness and attitude regarding HIV/AIDS and high risk behaviour.

Methods Primary data has been collected from selected garment unit in Mumbai city. In-depth interviews and focus group discussion have been conducted using semi structured questionnaire to among women garment worker in Mumbai.

Results Interviews with migrant garment workers confirmed that poverty was the primary motivator for migration. Women and key informants reported awareness that some migrants had sexual relationships with local men or engaged in sex work to supplement their income. Factory restrictions limited women's ability to access health care services and health education programs. Majority of the women had heard about HIV/AIDS and knew some aspects of it; the knowledge about it was very sparse. Migrant women and adolescent girls are more vulnerable to multiple partner sexual behaviour may be due to many reasons like poverty, harassment etc. Women involving in multiple partner sexual relations, which put them at greater health risk like RTI/STI/HIV.

Conclusion It is really a matter of concern as these women are highly vulnerable and can easily be infected with the HIV, considering the fact that they are not well aware about safe sexual practices. Adequate measures needs to be taken at various levels to address this issue more seriously.

\section{P4.86 WORKPLACE AND HIV-RELATED SEXUAL BEHAVIOURS AND PERCEPTIONS AMONG FEMALE MIGRANT WORKERS: A STUDY OF MUMBAI CITY}

Prashika. International Institution for Population Sciences, Mumbai, India

\subsection{6/sextrans-2017-053264.581}

Introduction HIV/AIDS has become a major public health problem in India. Its prevalence among young people aged 15-24 years is high. Migrant workers have been identified as a population at risk for acquiring and transmitting HIV in many countries. For several reasons female migrants are believed to be more vulnerable than their male counterparts. Several studies have established that high risk sexual behaviours are related to the working environment. So it is important to identify the relationship between the workplace and HIV risk this may help researchers to develop appropriately targeted prevention intervention strategies among this vulnerable subpopulation. To evaluate awareness and attitude regarding HIV/AIDS and high risk behaviour related to HIV infection among garment workers.

Methods Primary data has been collected from selected garment unit in Mumbai city. In-depth interviews and focus group discussion have been conducted using semi structured questionnaire among women garment worker in Mumbai.

Results Interviews with migrant garment workers confirmed that poverty was the primary motivator for migration. Mostly migrated to the urban settlements from rural areas. This has significantly changed their socio-economic behaviour and livelihoods; They mostly belong to diversified poor socio-economic background; The workers are very young, having poor knowledge of personal hygiene, sexual and reproductive health, safer sex practices, STI and HIV/AIDS. They have limited access to reproductive and sexual health care and preventive measures. Factory restrictions limited women's ability to access health care services and health education programs. Women involving in multiple partner sexual relations, which put them at greater health risk like $\mathrm{RTI} / \mathrm{STI} / \mathrm{HIV}$.

Conclusion It is really a matter of concern as these women are highly vulnerable and can easily be infected with the HIV, considering the fact that they are not well aware about safe 
sexual practices. Adequate measures needs to be taken at various levels to address this issue more seriously.

\section{P4.87 SHOULD WE BE USING TECHNOLOGY FOR PARTNER SERVICES?}

${ }^{1}$ Rachel E Kachur, ${ }^{1} \mathrm{~A}$ Caccamo, ${ }^{2} \mathrm{FV}$ Strona, ${ }^{1} \mathrm{~J}$ Kinsey, ${ }^{1} \mathrm{D}$ Collins,. ${ }^{1}$ Centres for Disease Control and Prevention, Atlanta, USA; ${ }^{2}$ Centres for Disease Control and Prevention, San Francisco, USA

\subsection{6/sextrans-2017-053264.582}

Introduction Since 2000, health departments throughout the US have used new technologies, such as the internet and mobile phones, to enhance the provision of services to persons with a sexually transmitted infection, including HIV, and their sex partners, also known as partner services (PS). This study reviewed the published literature to assess changes in partner service outcomes as a result of using technology for PS (tPS) and to calculate cost savings through cases averted.

Methods A systematic literature review of all US studies assessing tPS was conducted in June 2016. Outcome measures were captured and cost savings were calculated, when data were available.

Results Eight studies were identified, published between the years of 2000-2015. The most frequently used measures to evaluate tPS included the number of partners notified, evaluated, screened or tested; and new infections identified. Percentage of partners notified using technology ranged from $17 \%-64 \%$ and percentage evaluated ranged from $18 \%-26 \%$. Number of new infections identified ranged from 2-19. Total costs saved through new cases averted ranged from US $\$ 21120$ to US\$42 223. Where calculated, percentage of partners who otherwise would not have been notified was $50 \%$. Texting resulted in more contacts $(77 \%, 69 \%, 41 \%$, $\mathrm{p}<0.0001$ ) and quicker median response times $(57.5 \mathrm{~min}$ 1 day) than traditional partner services or using the internet.

Conclusion Data and outcome measures across the studies were not standardised, making it difficult to make generalizable conclusions. Where tPS was used, programs found increases in the number of partners notified, including those who otherwise would not have been notified, tested and treated. New infections were also identified. Improved response times and time to treatment were also seen as was re-engagement into care for previous HIV positive patients. Although not a replacement for traditional PS, tPS enhances PS outcomes.

\section{P4.88 WHEN KNOWING IS NOT ENOUGH TO PREVENT: RISK AND INFORMATION SUCH AS YOUNG ACTIVISM IN HIVI AIDS}

Regina Célia de Oliveira Bueno; Elaine Rabello. Instituto de Medicina Social - UERJ, Rio de Janeiro - RJ, Brazil

\subsection{6/sextrans-2017-053264.583}

Introduction Understand the circularity of information and its role or relation with the advancement of the HIV/AIDS epidemic among young people in Brazil; Discuss the role of knowledge in this context of great access and circulation of information, especially among young people.

Methods From authors such as Giddens, Casttels, Spink and Goffman, we discuss the circularity of networked knowledge and its implications and influence on the behaviour of young people in vulnerability. From the theoretical discussion, interviews were conducted with $8 \mathrm{HIV}$ positive and seronegative youths with the objective of identifying the sources of information they access about HIV/AIDS, in order to answer the following questions: What knowledge circulates? Where do they come from? Where are they going? How do they circulate? What is the purpose? What is the real impact of the information? What subjects add to it? Subsequently, the interviews were transcribed and submitted to the Thematic Content Analysis.

Results We identified three themes addressed by young people when questioned about information and knowledge they have about HIV/AIDS: the exchange of information in network media (internet, smarthphones) as the main way of circulating knowledge; The discourse of risk and its weight in the decisions taken (whether they are to "risk" or "protect"); And the idea of vulnerable youth and youth empowered by accessible and available information. Knowing is not enough to prevent, because several other factors interfere in the decision to adopt a protective attitude towards the infection. Information can also contribute to the inverse effect; by the sense of empowerment it gives the youth.

Conclusion It is necessary to understand, create and/or identify new ways of "taking care", that consider the dynamics of the circularity of knowledge and undo the myth that "knowing is sufficient to prevent", from the identification of other factors circulating in the dynamics.

\section{P4.89 GENDER AND THE AIDS EPIDEMIC IN ITS FOURTH DECADE: ARE THE SCIENTIFIC KNOWLEDGE INCREASING THE QUALITY OF SEXUAL LIFE OF WOMEN LIVING WITH HIV?}

${ }^{1}$ Regina Maria Barbosa, ${ }^{2}$ Cristiane S Cabral, ${ }^{3}$ Adriana A Pinho. ${ }^{1}$ NEPO/UNICAMP, Campinas - SP, Brazil; ${ }^{2}$ Faculdade de Saúde Pública/USP, São Paulo - SP, Brazil; ${ }^{3}$ IOC/FIOCRUZ, Rio De Janeiro-RJ, Brazil

\subsection{6/sextrans-2017-053264.584}

Introduction Since the beginning of the AIDS epidemics, the prevention of MTCT has imprinted the directions of the debates on women's sexual and reproductive rights. The emphasis on the use of condom as the unique way of preventing sexual transmission, together with gender/sexual norms, has undermined women's ability to make sense of the latest scientific knowledge on their own benefit. This paper seeks to shed light on this issue by analysing the relationship between the acquisition of up-to-date information regarding HIV prevention and the use of condom.

Methods A cross-sectional study (GENIH study) was conducted between 2013 and 2014 in São Paulo with a probabilistic sample of 975 WLHIV aged 18 to 49 . We applied weighted descriptive techniques and logistic regression to identify factors associated with the knowledge of new forms of HIV prevention among WLHIV such as the relationship between viral load (VL) and HIV transmission, and with condom use.

Results Although the great majority of women have correct information on the effects of HAART on VL and CD4 count, 